\title{
Over 10\% Gain of Output Power of Medium-Sized Solar Cells by an Improvement of Geometry of Collector Electrodes
}

\author{
Kei Obinata, ${ }^{1}$ Yasuhiko Takeda, ${ }^{1}$ Naohiko Kato, ${ }^{1}$ and Tomoyoshi Motohiro ${ }^{1,2}$ \\ ${ }^{1}$ Toyota Central Research and Development Laboratories, Inc., 41-1 Yokomichi, Aichi, Nagakute 480-1192, Japan \\ ${ }^{2}$ Green Mobility Collaborative Research Center, Nagoya University, Furo-cho, Chikusa-ku, Nagoya 464-8603, Japan \\ Correspondence should be addressed to Tomoyoshi Motohiro; motohiro@mosk.tytlabs.co.jp
}

Received 22 March 2013; Accepted 24 June 2013

Academic Editor: Wayne A. Anderson

Copyright (c) 2013 Kei Obinata et al. This is an open access article distributed under the Creative Commons Attribution License, which permits unrestricted use, distribution, and reproduction in any medium, provided the original work is properly cited.

\begin{abstract}
Practical solar cells require larger active areas than ones in the R\&D stage in laboratories. However, the enlargement lowers conversion efficiency because of the following two reasons: (1) increase of resistive loss in the transparent conductive electrode caused by the longer distance for the photogenerated carriers to pass and (2) active area loss caused by shadows of collector electrodes laid on to reduce the previous resistive loss. In the present study, we have constructed an advisable modeling method to calculate output power of medium-sized solar cells and optimized geometry of the collector electrodes. Results of the modeling taught us a possible improvement of the output power by over $10 \%$. These simulated results were reproduced by electrochemical experiments.
\end{abstract}

\section{Introduction}

Practical solar cells require large active areas than ones in the R\&D stage. Scaleup of solar cells from laboratory samples to practical modules usually decreases conversion efficiency, as evidently displayed by Green et al. [1] because of two inevitable reasons: (1) increase of resistive loss in the transparent conductive electrode caused by the longer distance for the photogenerated carriers to pass and (2) active area loss caused by shadows of collector electrodes laid on to reduce the previous resistive loss.

Although some radial patterns of collector electrode have been proposed in place of conventional parallel type to reduce the shadowing loss [2], the improvement has been reported to be as low as $2 \%$, accompanied by the disadvantage of higher cost caused by the necessity to form a through-hole at the center of the radial pattern to realize back contact.

The adverse influence of collector electrodes on dyesensitized solar cells (DSSCs) is more remarkable than that on $\mathrm{Si}$ solar cells, because protection layers such as a glass flit passivation or a bismuth borosilicate-based thick film passivation $[3,4]$ over the silver wires is necessary to prevent the silver wires from corrosion by the electrolyte. Since the area shadowed by the protection layer as well as the collector electrode contributes to the loss of photovoltaic power, the active area further decreases in the case of DSSC compared with Si solar cells. This disadvantage of DSSCs, however, implies that the optimization of the geometry of the collector electrode should be more effective in the improvement of conversion efficiency than in Si solar cells.

Therefore, here, we developed the modeling of the geometry of the collector electrode to give the highest output power of medium-sized solar cells with a special attention to features of DSSCs.

In the present study, we have constructed an advisable modeling method for calculation of output power of medium-sized solar cells to optimize the geometry of the collector electrodes. We carried out electrochemical experiments to verify the effect of the optimized geometries.

\section{Simulations}

2.1. Structure of Solar Cells. Figure 1 displays a cross-sectional structure of a typical DSSC. The front-side electrode of DSSCs is a glass sheet coated with a conductive oxide (TCO) layer transparent for the visible sunlight. Since the sheet resistance 


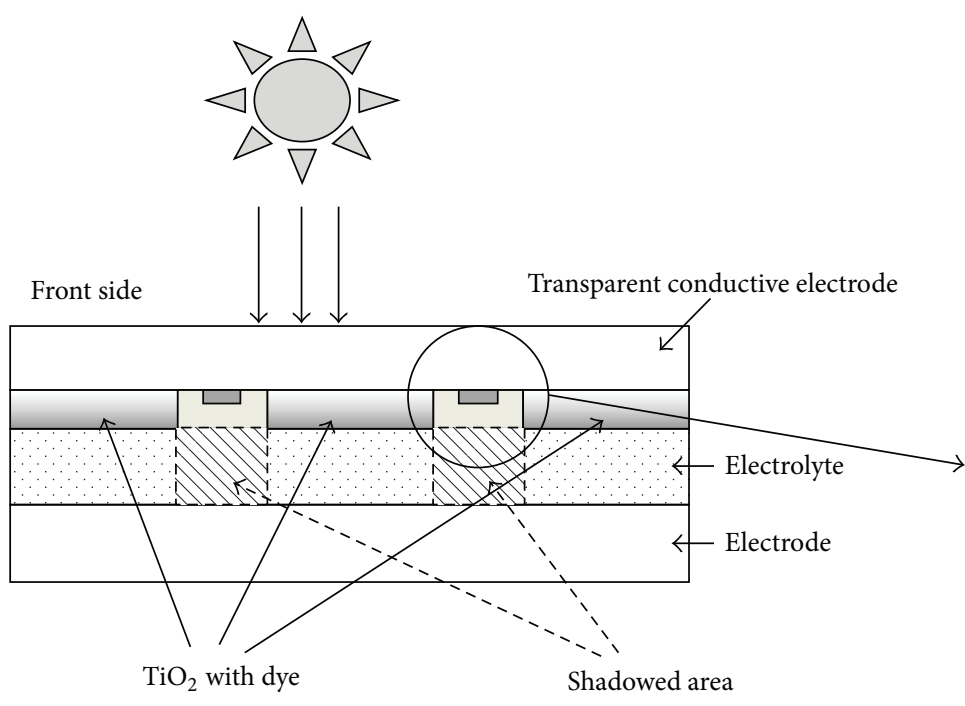

(a)

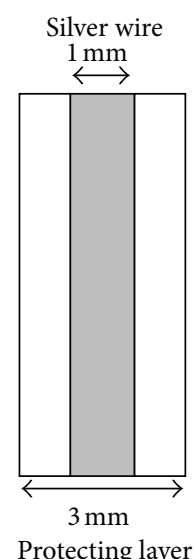

(b)

FIgURE 1: Schematic diagram of a dye-sensitized solar cell: (a) a side view, (b) an enlarged top view of a collector electrode portion.

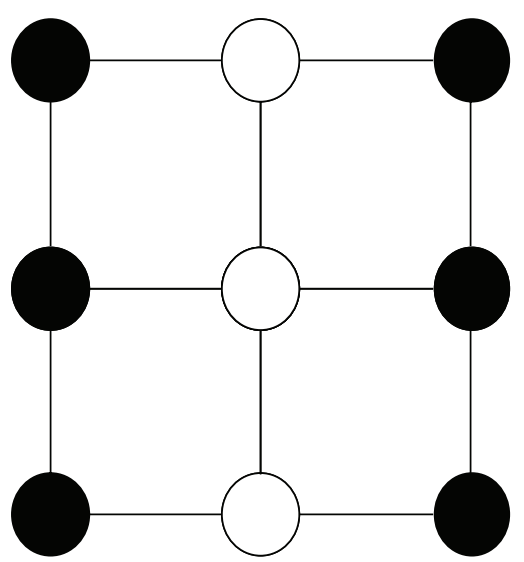

(a)

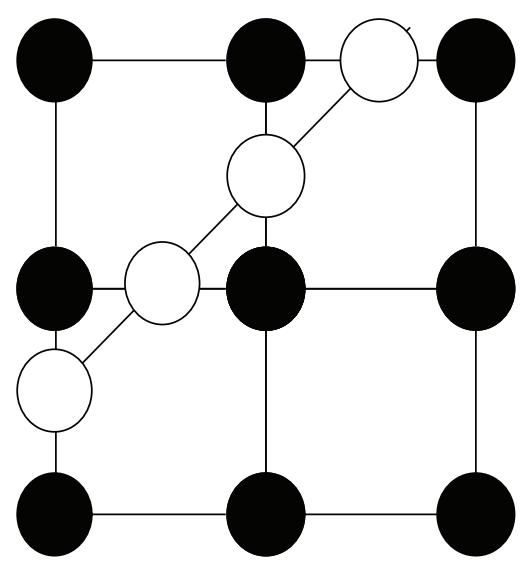

(b)

FIGURE 2: Division methods. Active nodes are indicated with closed circles and collector nodes indicated with open circles. Lines indicate links. (a) Equal division and (b) unequal division for collector electrodes.

of TCO layer around $10-20 \Omega / \square$ is not low enough if the size of the cells increases from around 1 square centimeter to hundreds of square centimeter, additional collector electrodes are necessary to decrease a resistive loss of the output power. The collector electrodes used for DSSCs consisting of silver wires with their cross-sectional size of typically $1 \mathrm{~mm}$ in width and 10 micrometers in height and protection layers of typically tens of micro-meters in thickness and $3 \mathrm{~mm}$ in width, covering silver wires completely, are screen-printed on TCO layers on glass substrates [5]. Consequently, the shadowed area of the width of $3 \mathrm{~mm}$ became nonactive for photovoltaic power.

2.2. Modeling Method. We evaluated the performance of solar cells with various collector electrode geometries using a newly devised 2-dimensional model. Solar cells are approximated to consist of elements referred to as "nodes" and "links" that connect the neighboring nodes. There are two kinds of nodes: active nodes and collector nodes representing the active areas and the collector electrodes, respectively.

The method to divide the whole cell into the elements is a great issue for compatibility of accuracy and reasonable computation time. Figure 2 shows two types of dividing methods. Equal division of a square arrangement illustrated in Figure 2(a) is efficient for active nodes and is also suitable for collector nodes when the collector electrodes are parallel and/or perpendicular to the edges of a square or rectangular cell. However, in order to represent collector electrodes in a slant direction accurately, an extremely large number of nodes are needed, resulting in an unrealistic long computation time. To avoid this, each element of equally divided cells was dealt with as an active node and connected by links, and collector nodes were set on the location of the collector electrodes. The effect of shadows of the electrodes 


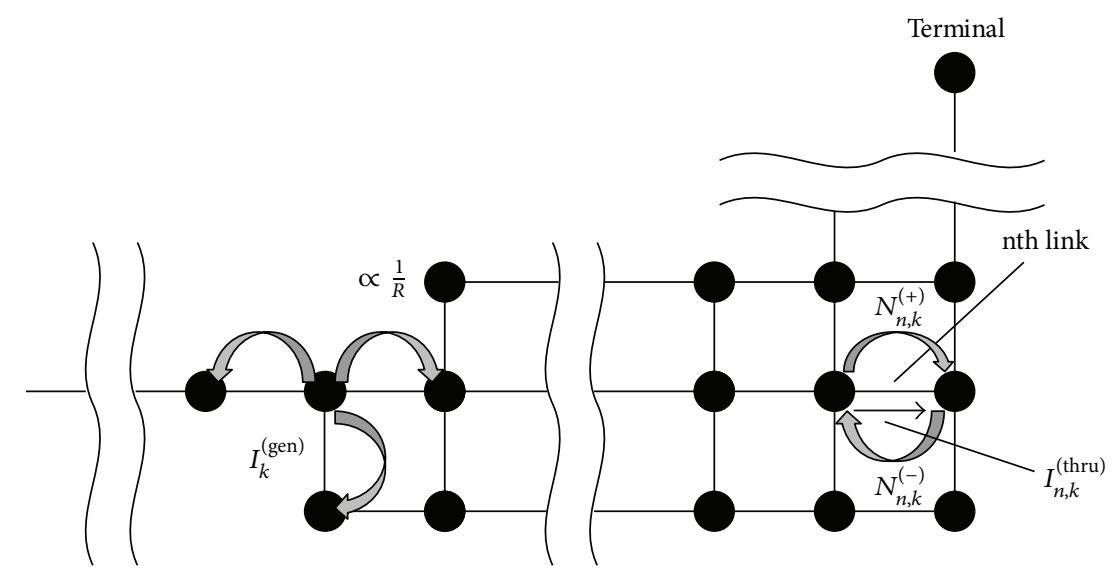

FIGURE 3: Schematic diagram of calculation of trajectories of $I_{k}^{\text {(gen) }}$ by random-walk method.

was involved by reducing the areas of the corresponding active nodes. Figure 2(b) depicts the arrangement of active and collector nodes connected by links employed here.

Let $I_{k}^{\text {(gen) }}$ be the current photogenerated at the $k$ th node. An active node is approximated to work at the maximal power point (MPP) of the solar cell operation to generate electricity, because the working point is only slightly different from the MPP in reality depending on the location in an efficient cell. Therefore, $I_{k}^{\text {(gen) }}$ is equal to the product of the current density at MPP, $J_{\max }$, and the area of the element represented by the $k$ th node. The locally generated power at the $k$ th node equals $V_{\max }$ (the voltage at MPP) times $I_{k}^{\text {(gen) }}$.

The assumption that all the active nodes work at the MPP allows us to deal with them as independently working ones. Therefore, as shown in Figure 3, the trajectory to reach the output terminal is sought using a random-walk method $[6,7]$ that has been originally developed for a single power source system. The probability that $I_{k}^{\text {(gen) }}$ flows to one of the neighboring nodes is inversely proportional to the resistance of the link connecting the two nodes. The probability for the next step is similarly evaluated. Thus, the contribution of $I_{k}^{(\text {gen) }}$ to the current passing through the $n$th link, $I_{n, k}^{\text {(thru) }}$, is expressed as follows:

$$
I_{n, k}^{(\text {thru })}=\sum_{T} \frac{I_{k}^{(\text {gen })} \times\left(N_{n, k}^{(+)}-N_{n, k}^{(-)}\right)}{T},
$$

where $N_{n, k}^{(+)}$is the number that $I_{k}^{(\text {gen) }}$ flows through the $n$th link in one direction before reaching the output terminal, and $N_{n, k}^{(-)}$is the number in the opposite direction, among the $T$ times trial of the random walk $(T=10000)$. The total current in the $n$th link, $I_{n}^{\text {(link) }}$, equals the summation of $I_{n, k}^{\text {(thru) }}$ over all the active nodes:

$$
I_{n}^{(\text {link })}=\sum_{k} I_{n, k}^{(\text {thru })}
$$

The output power of the cell, $P$, is lower than the summation of the generated power of all the nodes by the resistive loss. Thus, the expression of $P$ is derived as follows:

$$
P=V_{\max } \sum_{k} I_{k}^{\text {(gen) }}-\frac{1}{2} \sum_{n} R_{n}\left(I_{n}^{\text {(link) }}\right)^{2} .
$$

The value of the resistance of the $n$th link, $R_{n}$, connecting two neighboring active nodes is determined from the series resistance of a cell that is dominated by the resistance of the TCO used in the cell, considering the fact that the $R_{n}$ values are inversely proportional to the length of the links to which the nodes on both ends correspond.

The $R_{n}$ between two collector nodes is exactly the resistance of the collector electrodes proportional to the length of the link, that is, center-to-center distance between the corresponding two nodes. When a link connects an active node and a collector node, $R_{n}$ is dominated by the resistance of TCO at the active node, which is larger than the resistance of the collector electrode by three-order of magnitude.

We used the $J_{\max }, V_{\max }$, and series resistance of a cell derived from previously reported results for a DSSC of $5 \mathrm{~mm} \times 5 \mathrm{~mm}$ in size (see $[8,9])$. To determine the value of $R_{n}$, we measured the resistance of screen-printed silver wires on the TCO layer used in the present experiments to be $0.23 \Omega$ at $10 \mu \mathrm{m}$ in thickness, $1 \mathrm{~mm}$ in width, and $10 \mathrm{~cm}$ in length. It should be noted that this measured value of $R_{n}$ includes conductance of TCO as well as silver paste itself.

The first term in (3) is proportional to $I_{k}^{\text {(gen) }}$, whereas the second term is proportional to the square of $I_{k}^{\text {(gen) }}$.

Therefore, $P$ exhibits a maximum somewhere with increasing the incident irradiation intensity. This does not take place in the region of the present concern, because the dependence of $V_{\max }$ on the incident irradiation is minor, and $R_{n}$ is constant.

We verified the validity of (3). In the case where light irradiate solar cells uniformly, the current $I_{n}^{\text {(link) }}$ is proportional to generation current $I_{k}^{\text {(gen) }}$. Suppose the current used in the resistive loss estimation in the second term is 


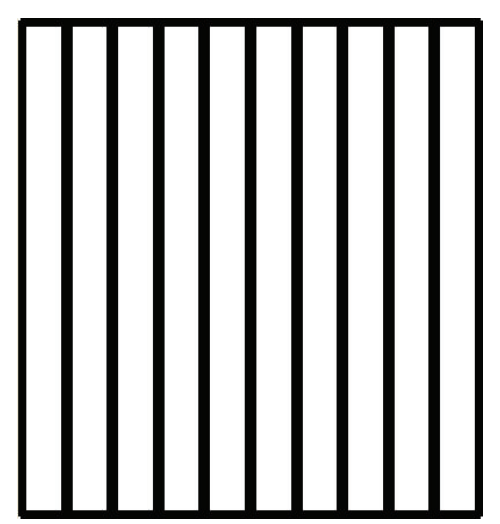

(a)

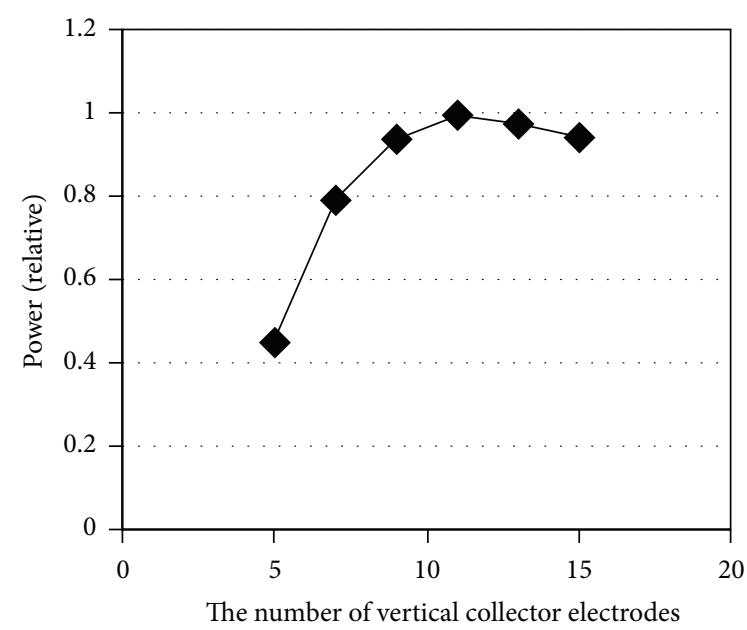

(b)

FIGURE 4: Geometries of standard collector electrodes and their relative power: (a) Standard geometry, (b) relative power changing with the number of vertical electrodes.

the total current, and the resistivity is also supposed to be its maximum value, (3) can be rewritten as follows:

$$
\begin{gathered}
P=V_{\text {max }} I_{\text {total }}-\frac{1}{2} R_{\max } I_{\text {total }}^{2}, \\
I_{\text {total }}=\sum_{k} I_{k}^{\text {(gen) }} .
\end{gathered}
$$

We estimated current, resistance, and voltage in reasonable condition. In usual conditions under the 1 sun $\left(100 \mathrm{~mW} / \mathrm{cm}^{2}\right)$ insolation, DSSCs generally yield around $20 \mathrm{~mA} / \mathrm{cm}^{2}$ or lower. This means that $I_{\text {total }}$ is around or lower than $2 \mathrm{~A}$ in the present cell of around $10 \mathrm{~cm} \times 10 \mathrm{~cm}$ in size. For resistance, in the present concern, it is hard to consider that $R_{\max }$ exceeds $50 \Omega$ since sheet resistance of TCO is about $10 \Omega / \square$.

As for voltage, we have also confirmed that the voltage drops in the links between the collector nodes are negligibly small, experimentally. The estimated voltage drop was at most $10 \mathrm{mV}$, that is, around $2 \%$ of $V_{\max }$ under the present conditions. Therefore, the approximation that all the nodes generate electricity equal to $V_{\max } \times I_{k}^{\text {(gen) }}$ can be used.

The present method to calculate $P$ is based on the approximation of photogenerated current uniformly distributed like the method of Burgers [10]. Therefore, the resultant $P$ value by solving an equation representing an electric circuit would be accurate. Although more efficient methods to solve the equation have been developed by Bissels et al. [11] and Zhang et al. [12], they can be used only for simple geometries to find stable solutions.

In contrast, the present method can be applied to complicated geometries like being examined here with sufficiently high accuracy. The computational complexity of the present method, however, is not suitable for calculation on extreme conditions such as solar cells of very low photovoltaic performance or high resistance as written later.
2.3. Geometry of Collector Electrodes. At the start of the series of simulations, we calculated output power of a 2-dimensional "Standard geometry" of the collector electrode generally used for DSSCs as shown in Figure 4(a). The number of the vertical collector electrodes has been optimized to be 11 to yield the maximum power in the simulation as shown in Figure 4(b). We rated the performance of new geometries of the collector electrode by comparing the output powers with that of Standard geometry. We tried varieties of geometries of the collector electrodes, but almost all the new geometries showed poorer performance compared with Standard geometry. Figure 5 shows some examples of the poorer performance geometries for reference.

As an exception, the geometry in Figure 6(a) gave almost a similar performance to the Standard geometry. However, it is apparently much more difficult for actual fabrication because of its complicated pattern. As for another exception, the geometry in Figure 6(b) gave a 10\% better performance. However, it is based on the connection of a terminal electrode at the center of the pattern which is not feasible in reality. This geometry is practically possible, for example, by using a via hole for the output terminals [13] but would increase production cost.

Figure 7 shows the current distribution of the geometry of electrodes: a group of parallel straight electrodes connected with each other at the one side. The current in the collector electrodes located close to the output terminal electrodes on the top edge is remarkably large, resulting in serious resistive loss. To avoid this current concentration, we devised several geometries using slanting collector electrodes. Geometry 2 illustrated in Figure 8(b) is expected to have the electrical resistance comparable to Standard geometry with a larger active area. Geometry 3 and Geometry 4 shown in Figures 8 (c) and $8(\mathrm{~d})$ are to provide lower electrical resistance with a small loss of active area. Geometry 3 in Figure 8(c) has variation for locating terminal point of slanting collector electrodes. In the example shown in Figure 8(c), the terminal 


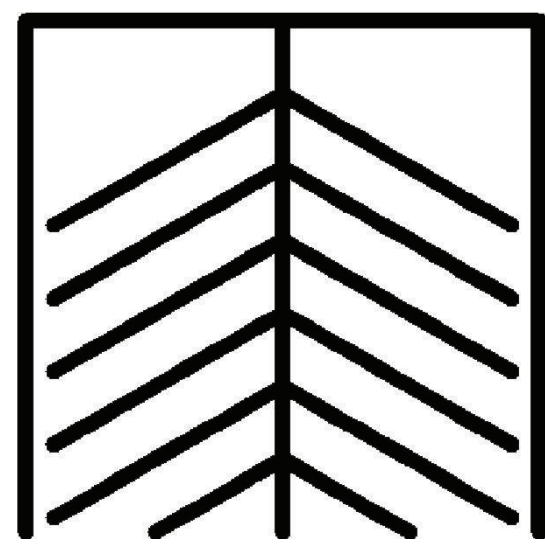

(a)

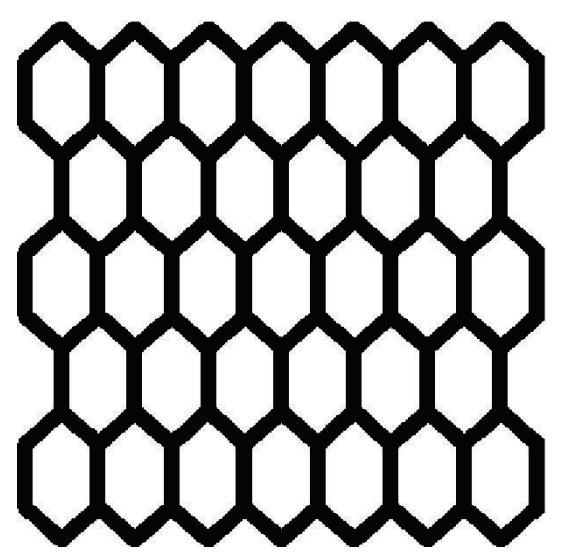

(b)

FIGURE 5: Geometries of poorer performance than the Standard geometry in Figure 4. (a) Nerve type and (b) honeycomb type.

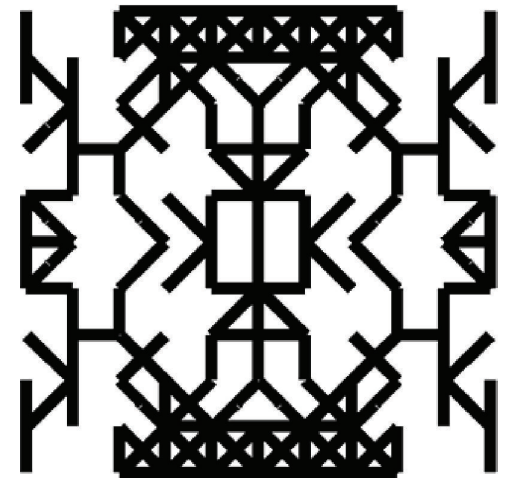

(a)

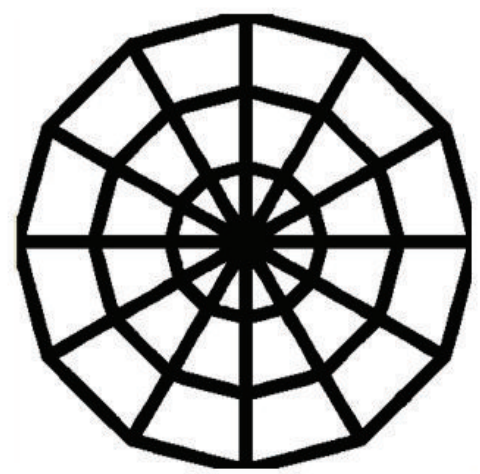

(b)

FIGURE 6: Examples of geometries of good performance with problem in fabrication. (a) The pattern produced by a genetic algorithm. (b) The pattern that has an output terminal in the center.

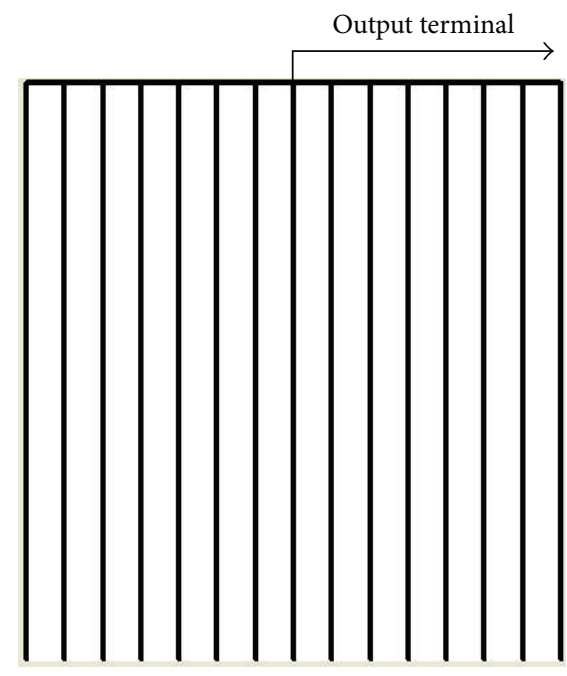

(a)

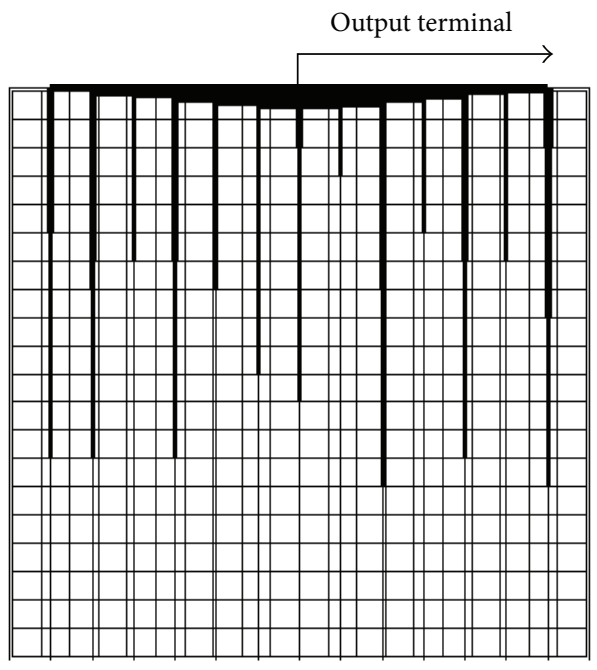

(b)

FIGURE 7: (a) A finger electrode geometry. (b) Current density in the electrodes. Thickness of line is proportional to the current density. 


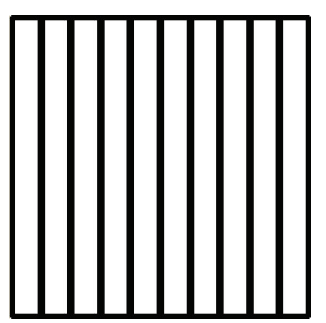

(a)

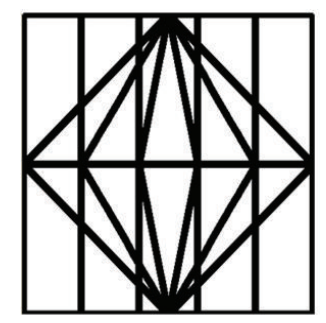

(b)

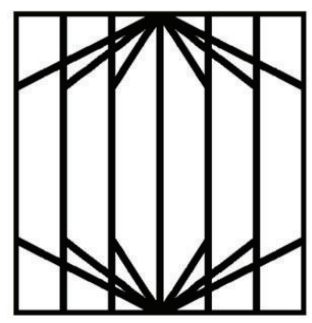

(c)

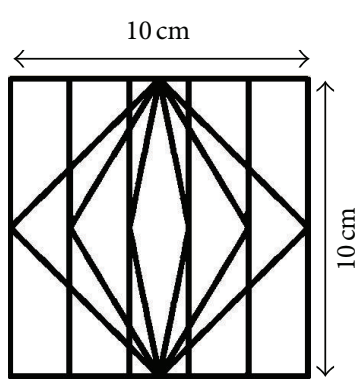

(d)

Figure 8: Devised geometries (a) Standard geometry, (b) Geometry 2, (c) Geometry 3, and (d) Geometry 4. The output terminal is supposed to be located at the same position as in Figure 7.

points are chosen to locate at $2.5 \mathrm{~cm}$ from upper side of cell to give the best performance.

2.4. Simulated Results. Figures 9(a)-9(c) show calculated output power $P$ of the three types of geometries as a function of the number of the vertical collector electrodes in the direction perpendicular to the top edge. Significant improvement up to 1.2 times has been predicted by choosing an appropriate number of vertical collector electrodes for each geometry.

\section{Experiments}

3.1. Geometries of Collector Electrodes Used for Experimental Verification. From the experiences mentioned previously in chapters 2.3 and 2.4, we have learned the possibilities that the radial (c.f. Figure 6(b)) and slanting collector (c.f. Figure 9) electrodes can avoid current concentration on the collector electrodes close to the connecting terminals. Based on this, three types of geometries were devised as shown in Figures 10(b)-10(d). Figure 10(a) shows Standard geometry' for experiments. The reason why a "dash" attached to "geometry" is because a small protruded portion was newly laid on to the geometry of collector electrode in actual experimental procedure for ease of wiring to extract current. To give a space for the protrusion, the size of each pattern was changed from $10 \mathrm{~cm} \times 10 \mathrm{~cm}$ to $9.5 \mathrm{~cm} \times 10 \mathrm{~cm}$. Each geometry shown in Figures 10(a)-10(d) with "dash" corresponds to that of Figures 8(a)-8(d). Additional small changes were added to Geometries $3^{\prime}$ and $4^{\prime}$ so as to avoid formation of very small active area isolated with the collector electrodes.

3.2. Fabrication of Collector Electrodes. We fabricated collector electrode samples of the Standard geometry and the optimized ones based on the simulation, for experimental proof, although adjustment was added in production. The numbers of the vertical collector electrodes optimized as shown in Figure 9 by the simulation were adopted for Geometries $2^{\prime}$, $3^{\prime}$, and $4^{\prime}(5,7$, and 6 vertical collector electrodes, resp.).

An automatic printing machine was used for printing silver paste to form silver wires of typically $10 \mu \mathrm{m}$ in thickness and $1 \mathrm{~mm}$ in width on glass substrates covered with TCO (10 $\Omega / \square$, Nippon Sheet Glass). Then the samples were calcined at $450^{\circ} \mathrm{C}$. Finally, protection layers were printed over the silver wires. Silver wires on the TCO layer used in these experiments were confirmed experimentally to be $0.23 \Omega$ at $10 \mu \mathrm{m}$ in thickness and $10 \mathrm{~cm}$ in length after calcination as described in the Simulations section.

3.3. Experimental Evaluation Method. The samples consisting of TCO-covered glasses, silver wires, and protection layers were evaluated using a setup schematically illustrated in Figure 11. The sample was set as an anode in an electrolyte of $500 \mathrm{mM}$ sodium sulfate, with a copper plate as a cathode.

The protection layers prevented leak current from flowing out of the silver wires to the electrolyte, which takes place also in real DSSCs.

We used resin TB2249G (ThreeBond Co., Ltd.) for protection layer with the thickness of $500 \mu \mathrm{m}$. Widths of all the collector electrodes were set to $3 \mathrm{~mm}$ as in the simulation. The distance between the anode and cathode was set to be $5 \mathrm{~mm}$, which is $1 / 20$ of the sample size $(10 \mathrm{~cm} \times 10 \mathrm{~cm})$, so that the electrical resistance of the electrolysis solution was much larger than that of the samples, and consequently the whole of the sample area contributes to the current flow, like in real DSSCs.

In order to prevent adhesion of hydrogen and oxygen bubbles generated by water splitting to the electrodes, surfactant was added in the solution at a concentration of $1 \mathrm{~mL} / \mathrm{L}$; ultrasonic vibration was applied during the measurements.

Figure 12 shows the current-voltage relationship of the Standard geometry. Stable current larger than $1 \mathrm{~mA}$ was observed at an external voltage of $2.0 \mathrm{~V}$. Therefore, current values at 2.0,2.4, and $2.7 \mathrm{~V}$ were acquired for comparison of the fabricated geometries. The current values around $10 \mathrm{~mA}$ under these conditions are significantly lower than the output current in the simulation being around $500 \mathrm{~mA}$ under the 1 sun solar irradiation. Nonetheless, we employed these external voltage values, to minimize the change in the concentration of the electrolyte and to suppress adhesion of hydrogen and oxygen bubbles that remarkably disturb the current flow.

Just after an external voltage was applied, the current rapidly decreases, because the combination of an anode and a cathode with an electrolyte between them performs as a capacitor, as shown in Figure 13. After the first $60 \mathrm{~s}$, 

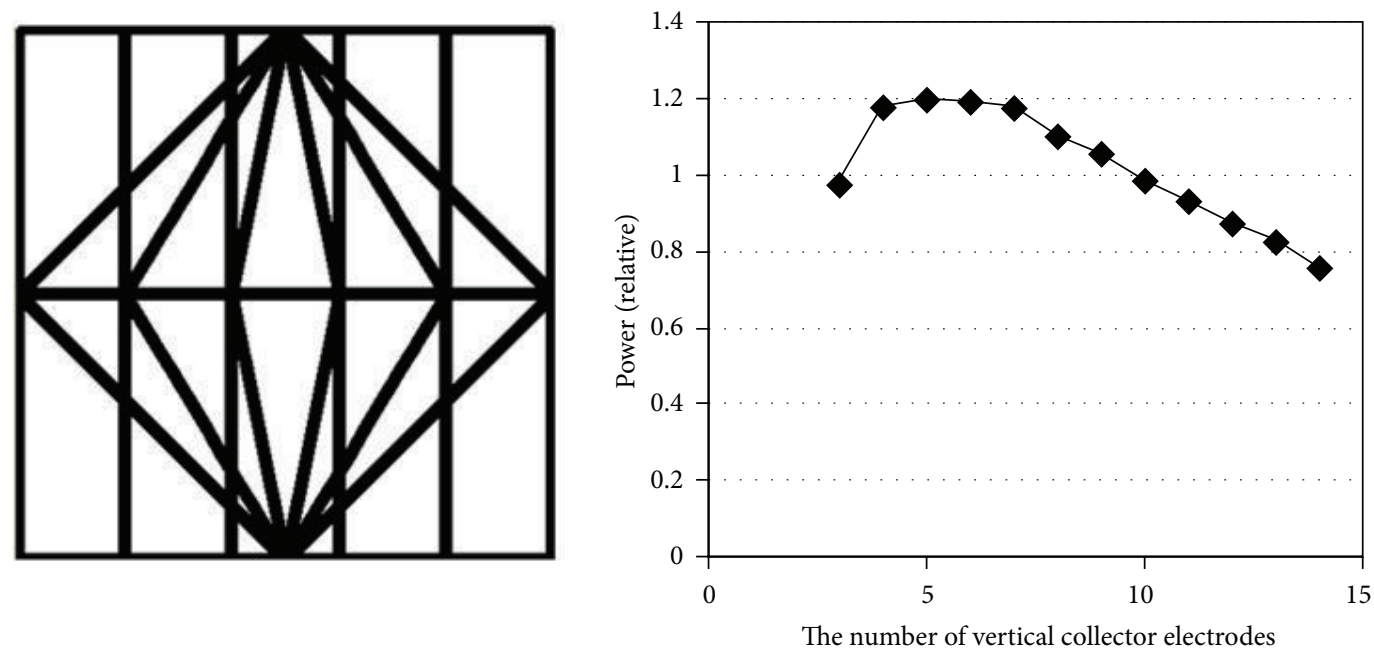

(a)
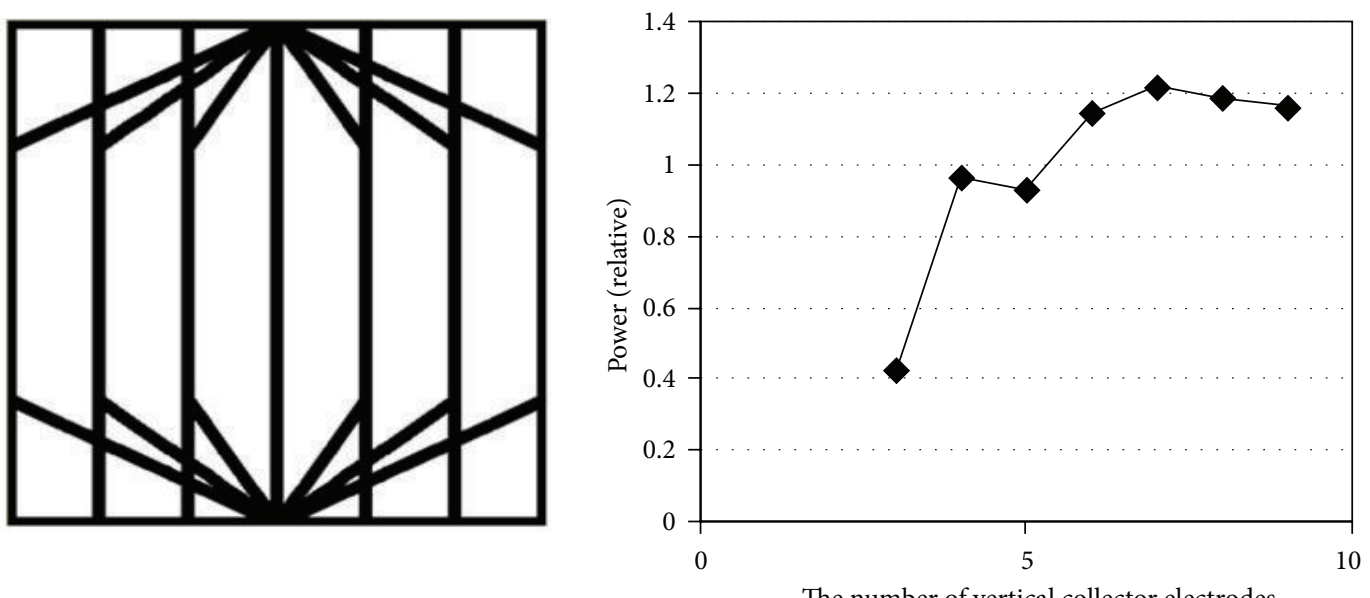

(b)
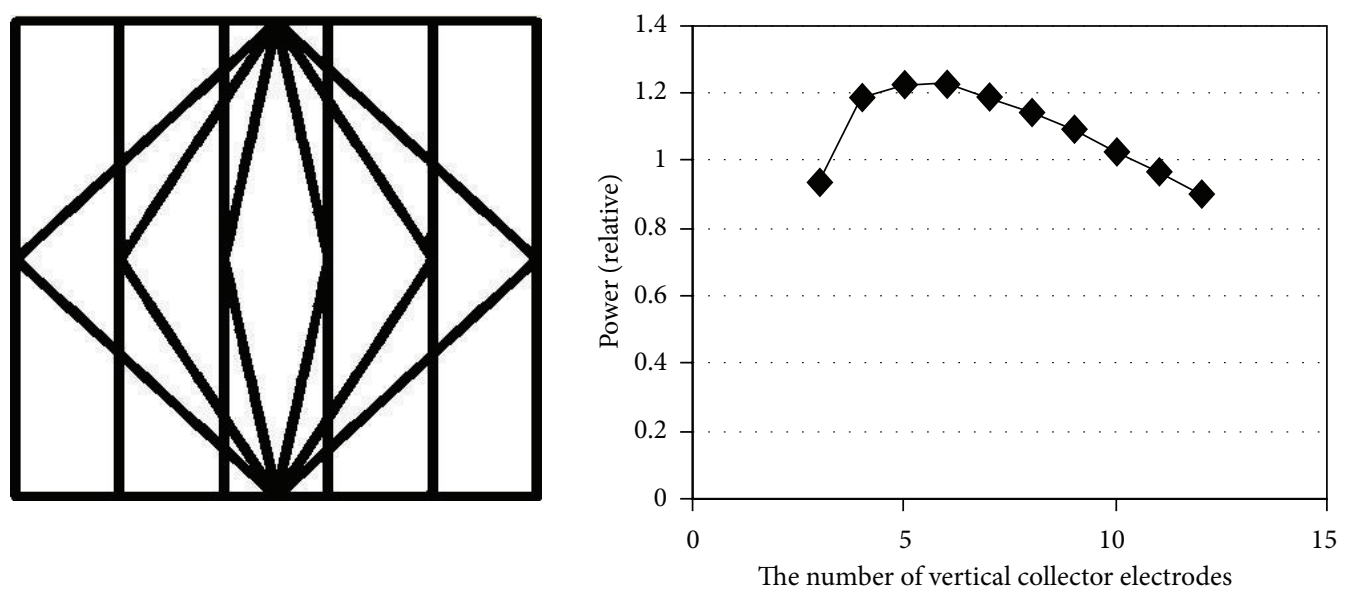

(c)

Figure 9: The optimized geometries and the dependence of the output power on the number of the vertical electrode for (a) Geometry 2, (b) Geometry 3, and (c) Geometry 4. 


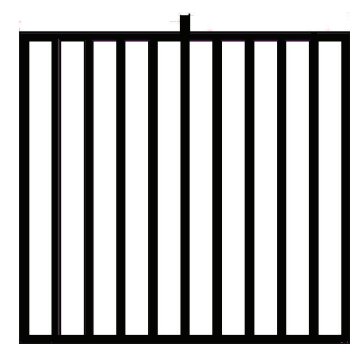

(a)

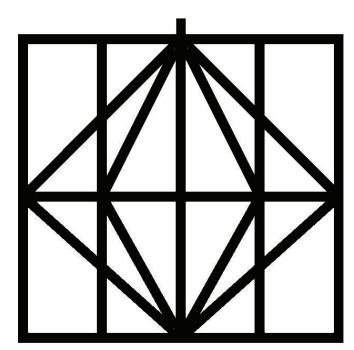

(b)

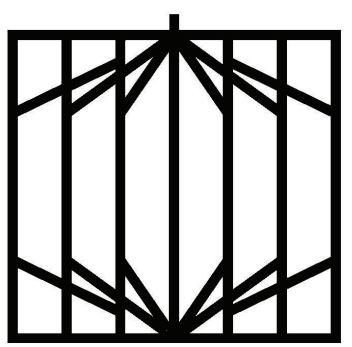

(c)

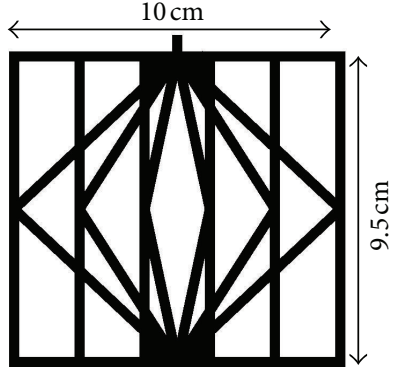

(d)

FIGURE 10: Geometries of collector electrodes used for actual experiments. (a) Standard geometry for experiments (Standard'), (b) Geometry $2^{\prime}$, (c) Geometry $3^{\prime}$, and (d) Geometry $4^{\prime}$.

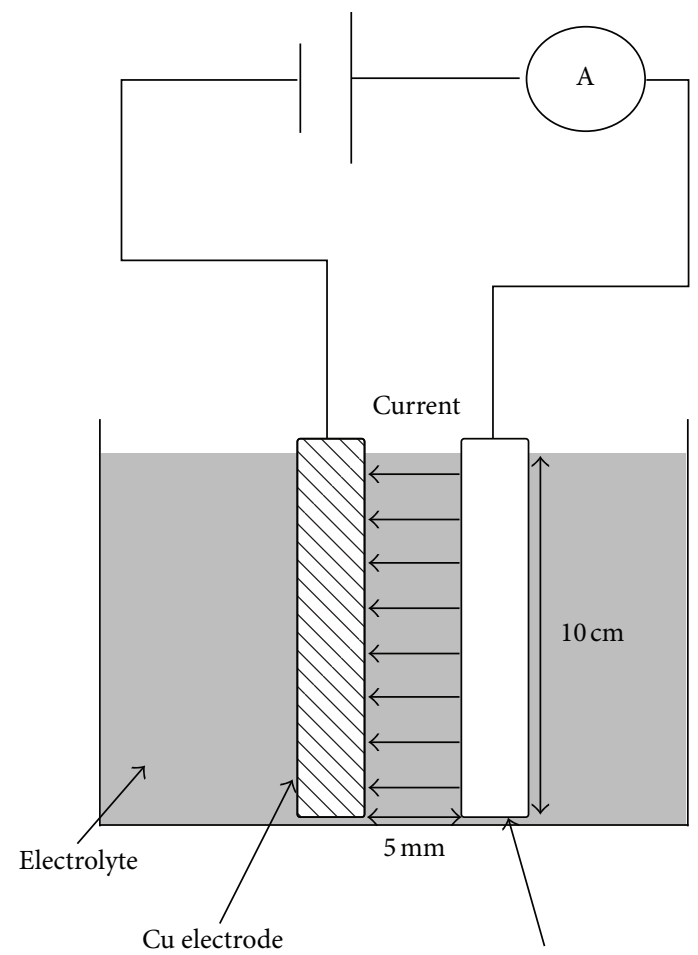

FIGURE 11: Schematic diagram of the electrochemistry experiments.

the decrease became small to reach a stabilized value for all the samples. Thus, current values after $120 \mathrm{~s}$ were adopted for evaluation.

3.4. Experimental Results. Figure 14 shows the measured value of the current for each geometry at $2.0 \mathrm{~V}, 2.4 \mathrm{~V}$, and $2.7 \mathrm{~V}$ of the external voltage. Geometry $2^{\prime}$ exhibited the largest current among the four kinds of the fabricated geometries. The values for Geometries $3^{\prime}$ and $4^{\prime}$ were also larger than that for the Standard geometry'.

In the present experiments, a larger active area generates a larger current value. However, when the resistance is larger, the voltage applied between the TCO and the copper plate more remarkably falls on an average, resulting in more significant lowering in the current value. Thus, the measured

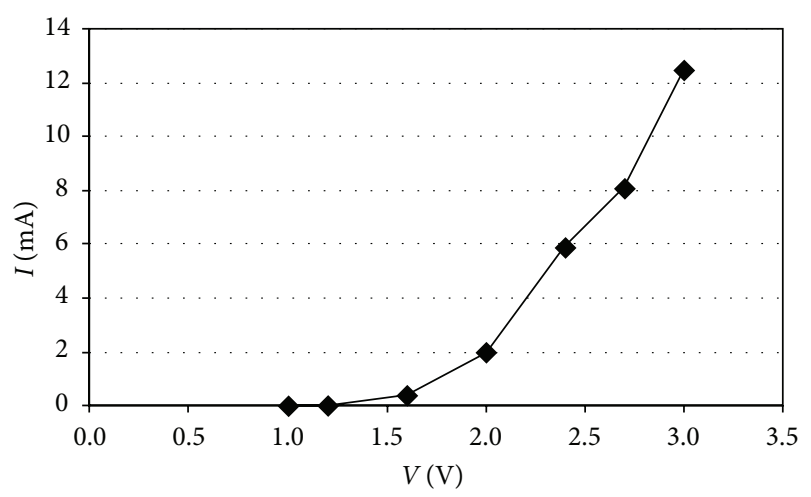

FIGURE 12: Current $(I)$-voltage $(V)$ curve of the Standard geometry.

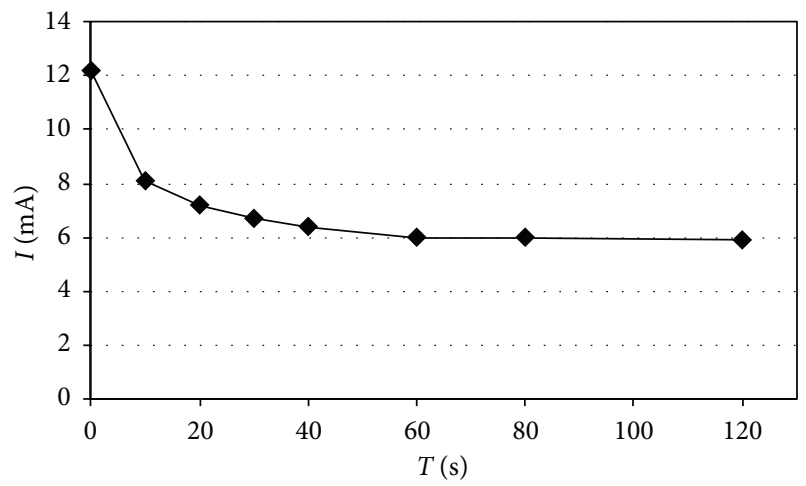

FIGURE 13: Temporal change in the current of the Standard geometry at $2.4 \mathrm{~V}$.

current value is equivalent to the performance of each geometry. Therefore, we can conclude that Geometries $2^{\prime}-4^{\prime}$ provide superior performance compared with the Standard geometry'. The gain achieved by the optimization of the collector electrode geometry was as high as $10-30 \%$.

3.5. Comparison of the Experimental and Simulated Results. Taking additional protrusion in experimental geometry of the collector electrodes into consideration, we carried out 


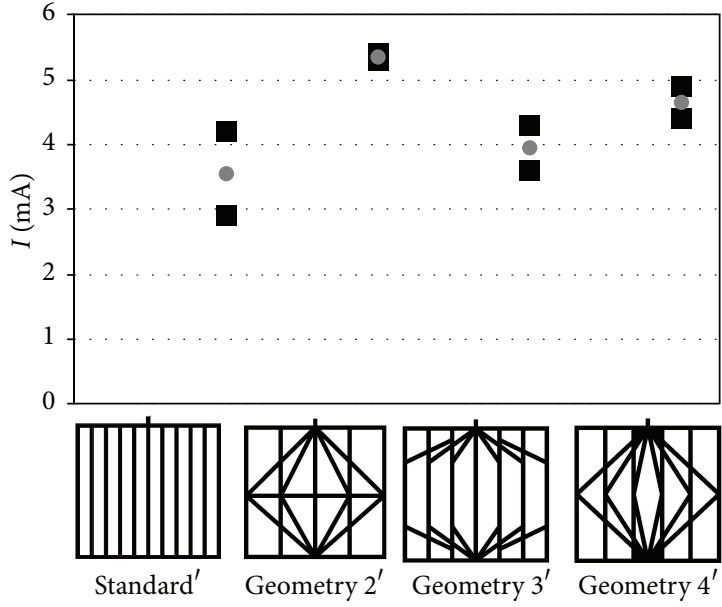

- One cell - Average

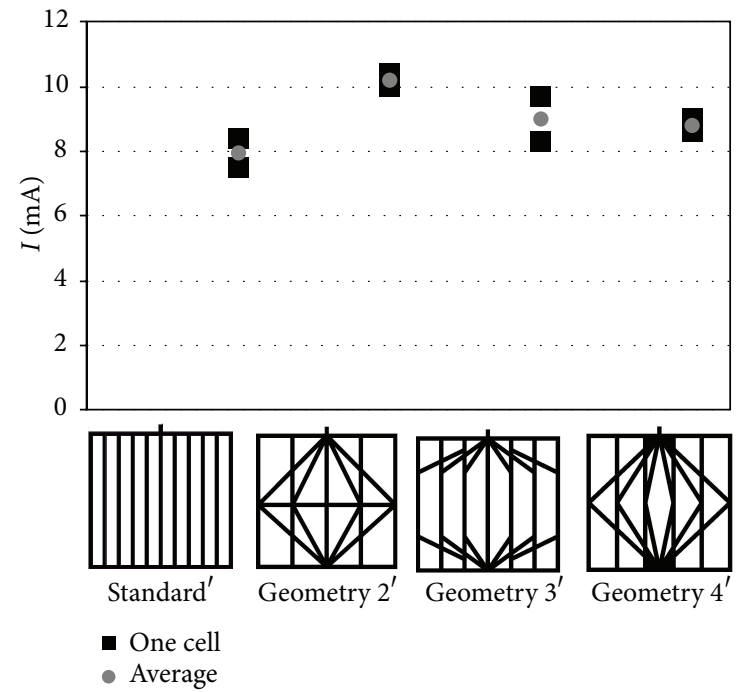

(b)

(a)

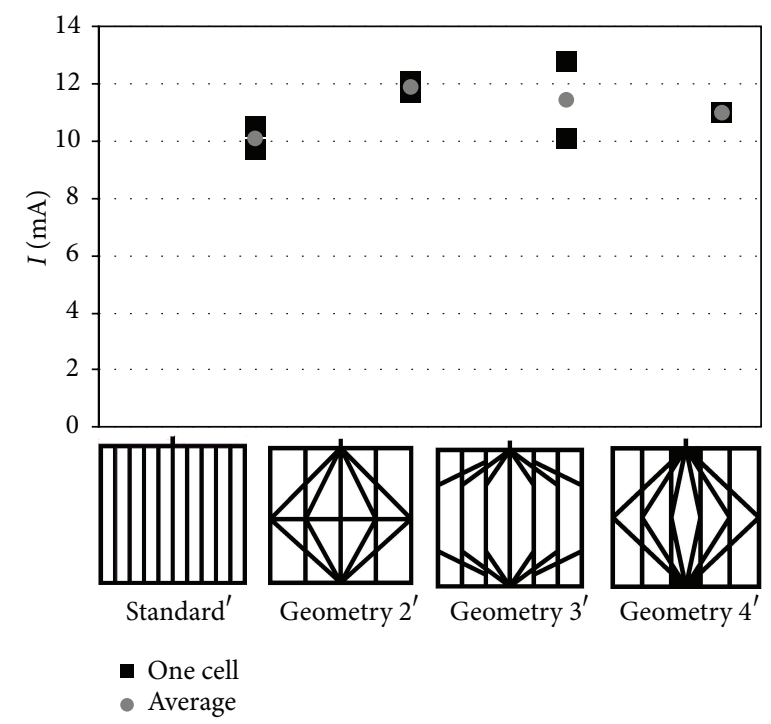

(c)

FigURE 14: Experimental results for the four geometries at (a) $\mathrm{V}=2.0 \mathrm{~V}$, (b) $\mathrm{V}=2.4 \mathrm{~V}$, and (c) $\mathrm{V}=2.7 \mathrm{~V}$.

simulations on geometries reproducing the experimental geometries more precisely which were necessary for the experimental proof. Figure 15 shows the new geometries employed here as well as the simulated results in black bars together with the corresponding experimental results. For Geometries $3^{\prime}$ and $4^{\prime}$, over $20 \%$ increase in output power relative to the value for the standard was expected, and this expectation almost agrees with the experimental values indicated in white bars. These improvements are due to reduction of the resistive loss.

In contrast, the output power decreases for Geometry $2^{\prime}$ because of an increase in the resistive loss. However, the experimental result for Geometry $2^{\prime}$ showed over $20 \%$ gain contrary to the expectation.

\section{Discussion}

The measured current values at $2.4 \mathrm{~V}$ are compared with the simulated output power values in Figure 15. The experimental results for Geometries $3^{\prime}$ and $4^{\prime}$ are in good agreement with the simulated ones. In other words, the predictions by the simulations have been successfully demonstrated.

Here, we must pay attention to the difference in the operating conditions. The simulation was carried out under the condition of 1 sun solar irradiation, with the resultant output currents being around $500 \mathrm{~mA}$. In contrast, the experiments were carried out with the current values of around $10 \mathrm{~mA}$ which corresponds to very low irradiation intensity so as not to yield bubbles on the surface of the electrodes. 


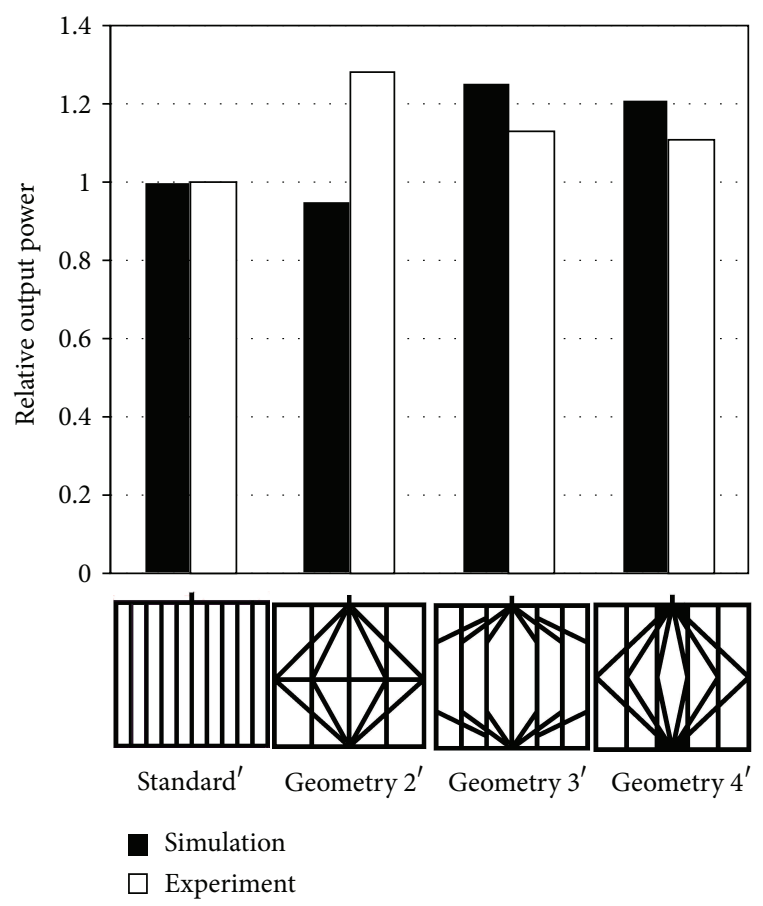

FIGURE 15: Comparison of the simulated and experimental results.

In the simulation, the output power goes up in the case of Geometry 2 in Figure 8(b) in comparison with Standard geometry in Figure 8(a). However, also in the simulation, the Geometry $2^{\prime}$ displayed in Figure 10(b) lost its output power in comparison with the Standard geometry ${ }^{\prime}$ in Figure 10(a). Here, again, it should be noted that Geometry $2^{\prime}$ is redesigned for experimental conveniences from Geometry 2 in Figure 8(a). Since only this geometry has larger active area than Standard geometry, it is considered that the influence of the decrease in the active area by having shortened length from $10 \mathrm{~cm}$ to $9.5 \mathrm{~cm}$ is greater than other geometries.

The reason for the difference between the results of experiment and those of simulation in Figure 15 is conjectured as follows.

The feature of large active area of Geometry 2 ' efficiently contributed to an increase in the current value. This geometry has a larger resistivity in comparison with other geometries. Since the resistive loss is proportional to the square of the output current, the impact of the resistive loss is less significant at lower irradiation intensity and a lower output current.

Therefore, under the experimental condition equivalent to weak irradiation, it is conjectured that the feature of large active area of Geometry $2^{\prime}$ efficiently contributed to an increase in the current value, whereas the other contributions such as of the relatively large resistance was small. According to this balance between the active area and resistive loss, it is conjectured that Geometry $2^{\prime}$ had higher output power in experiment. These features of Geometry $2^{\prime}$ are more suitable for indoor use, transparent cells with low absorbance, and so forth.
The present 2-dimensional simulation is applicable to the other types of solar cells with a transparent conductive electrode layer such as amorphous Si solar cells and other thin film solar cells. As for bulk silicon solar cells whether it is single crystalline or polycrystalline ones, it is better to use 3-dimensional simulation.

\section{Conclusion}

We have optimized geometry of collector electrodes for DSSCs using a newly constructed modeling to calculate output power of medium-sized solar cells. The simulation predicted that significant improvements in the output power over $10 \%$ are possible, relative to the Standard geometry. These results were reproduced by electrochemical experiments.

\section{Acknowledgment}

The authors thank Ms. Y. Uchiyama for her technical support in the fabrication of collector electrode samples.

\section{References}

[1] M. A. Green, K. Emery, Y. Hishikawa, W. Warta, and E. D. Dunlop, "Solar cell efficiency tables (version 39)," Progress in Photovoltaics, vol. 20, no. 1, pp. 12-20, 2012.

[2] J. H. Bultman, D. W. K. Eikelboom, R. Kinderman et al., "Fast and easy single step module assembly for back-contacted C-SI solar cells with conductive adhesives," in Proceddings of the $3 \mathrm{rd}$ World Conference on Photovoltaic Energy Conversion, pp. 979982, May 2003.

[3] W. J. Lee, E. Ramasamy, D. Y. Lee, and J. S. Song, "Glass frit overcoated silver grid lines for nano-crystalline dye sensitized solar cells," Journal of Photochemistry and Photobiology A, vol. 183, no. 1-2, pp. 133-137, 2006.

[4] D. H. Yeon, K. K. Kim, N. G. Park, and Y. S. Cho, "Bismuth borosilicate-based thick film passivation of Ag grid for largearea dye-sensitized solar cells," Journal of the American Ceramic Society, vol. 93, no. 6, pp. 1554-1556, 2010.

[5] E. Ramasamy, W. J. Lee, D. Y. Lee, and J. S. Song, "Portable, parallel grid dye-sensitized solar cell module prepared by screen printing," Journal of Power Sources, vol. 165, no. 1, pp. 446-449, 2007.

[6] A. Ghosh, S. Boyd, and A. Saberi, "Minimizing effective resistance of a graph," SIAM Review, vol. 50, no. 1, pp. 37-66, 2008.

[7] W. Ellens, F. M. Spieksma, P. Van Mieghem, A. Jamakovic, and R. E. Kooij, "Effective graph resistance," Linear Algebra and Its Applications, vol. 435, no. 10, pp. 2491-2506, 2011.

[8] K.-I. Ishibashi, Y. Kimura, and M. Niwano, "An extensively valid and stable method for derivation of all parameters of a solar cell from a single current-voltage characteristic," Journal of Applied Physics, vol. 103, no. 9, Article ID 094507, 2008.

[9] M. Murayama and T. Mori, "Equivalent circuit analysis of dye-sensitized solar cell by using one-diode model: effect of carboxylic acid treatment of $\mathrm{TiO}_{2}$ electrode," Japanese Journal of Applied Physics, Part 1, vol. 45, no. 1 B, pp. 542-545, 2006.

[10] A. R. Burgers, "How to design optimal metallization patterns for solar cells," Progress in Photovoltaics, vol. 7, pp. 457-461, 1999. 
[11] G. M. M. W. Bissels, M. A. H. Asselbergs, J. J. Schermer, E. J. Haverkamp, N. J. Smeenk, and E. Vlieg, "A genuine circular contact grid pattern for solar cells," Progress in Photovoltaics, vol. 19, no. 5, pp. 517-526, 2011, Erratum in Progress in Photovoltaics, vol. 20, pp. 994, 2011.

[12] Y.-D. Zhang, X.-M. Huang, K.-Y. Gao et al., "How to design dyesensitized solar cell modules," Solar Energy Materials and Solar Cells, vol. 95, no. 9, pp. 2564-2569, 2011.

[13] E. Van Kerschaver and G. Beaucarne, "Back-contact solar cells: a review," Progress in Photovoltaics, vol. 14, no. 2, pp. 107-123, 2006. 

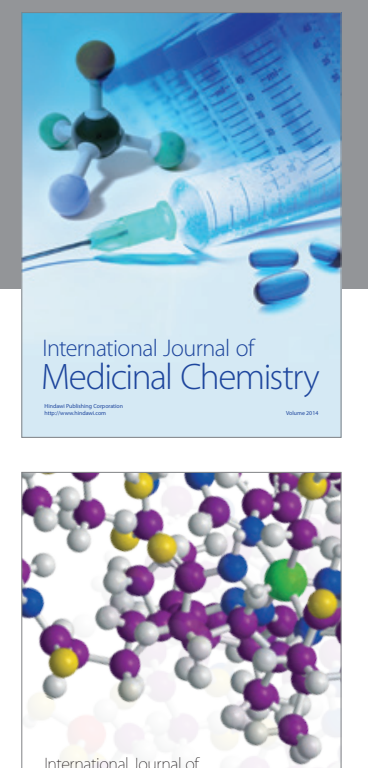

\section{Carbohydrate} Chemistry

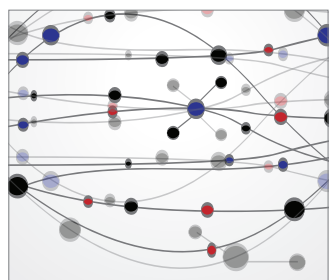

The Scientific World Journal
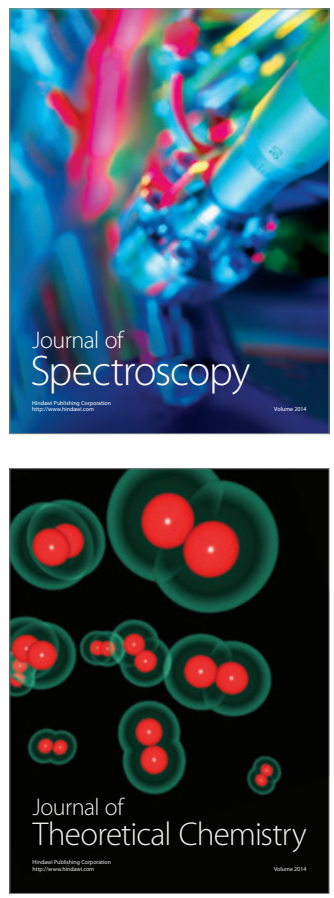
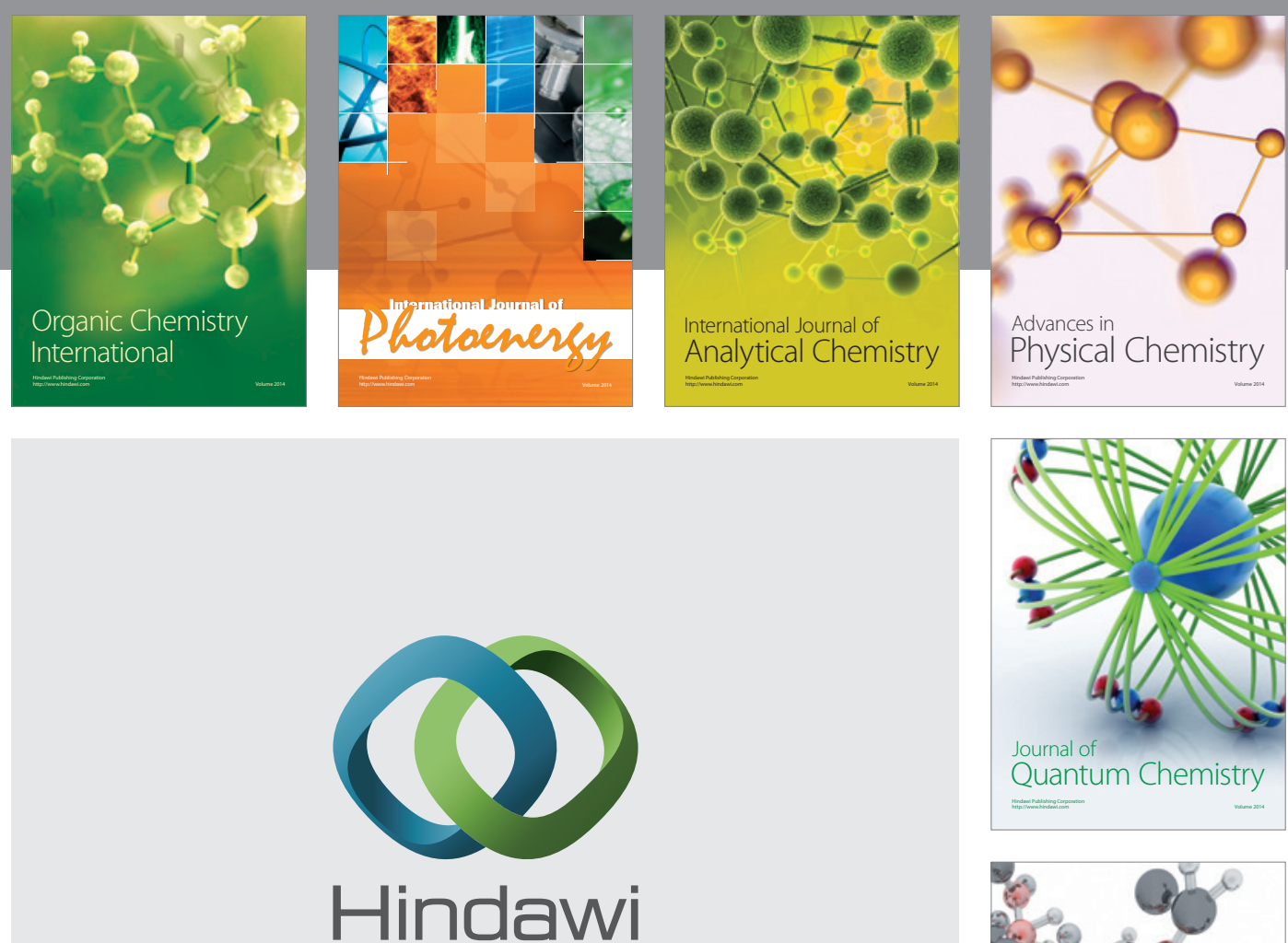

Submit your manuscripts at

http://www.hindawi.com

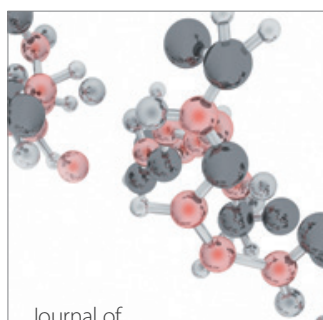

Analytical Methods

in Chemistry

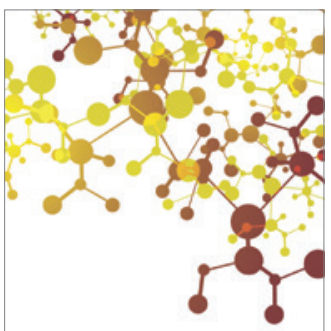

Journal of

Applied Chemistry

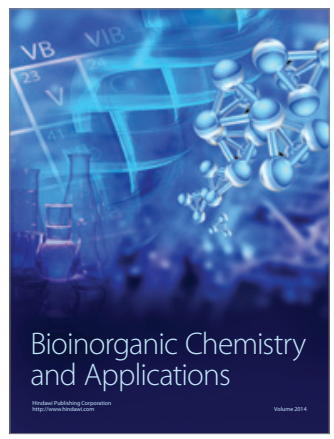

Inorganic Chemistry
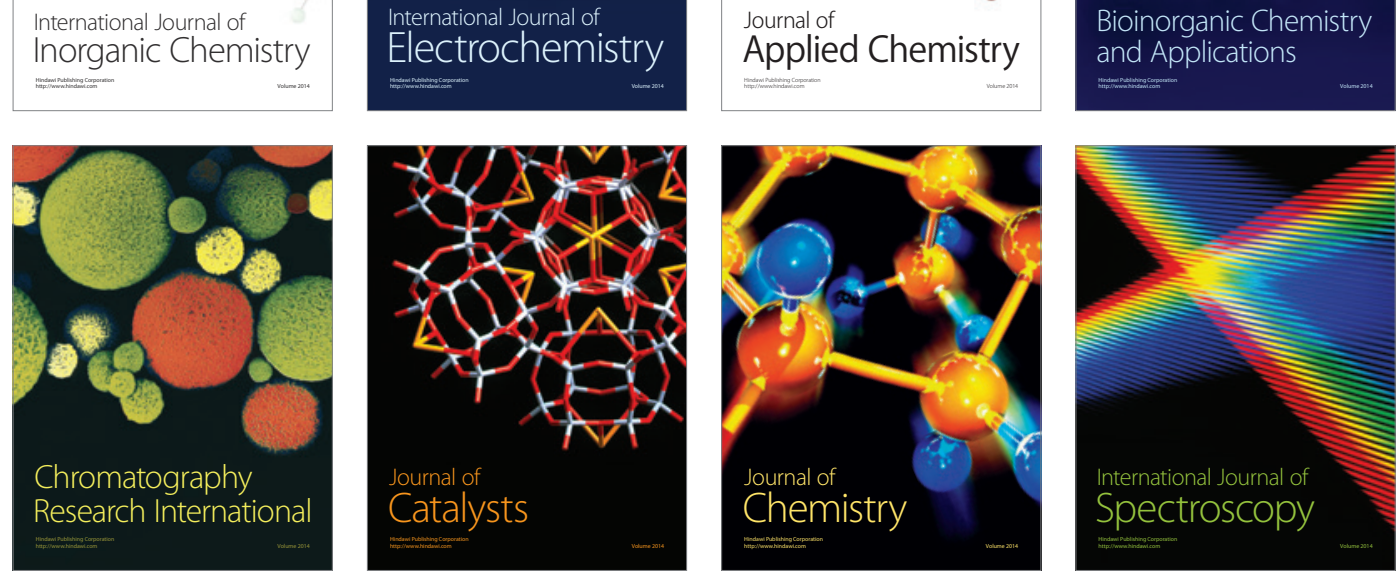